\title{
The effect of nanoparticle surfactant polarization on trapping depth of vegetable insulating oil-based nanofluids
}

\author{
Jian Li*, Bin Du, Feipeng Wang, Wei Yao, Shuhan Yao \\ State Key Laboratory of Power Transmission Equipment \& System Security and New Technology School \\ of Electrical Engineering, Chongqing University, Chongqing, 400044, China \\ E-mail: lijian@cqu.edu.cn
}

\begin{abstract}
Nanoparticles can generate charge carrier trapping and reduce the velocity of streamer development in insulating oils ultimately leading to an enhancement of the breakdown voltage of insulating oils. Vegetable insulating oil-based nanofluids with three sizes of monodispersed $\mathrm{Fe}_{3} \mathrm{O}_{4}$ nanoparticles were prepared and their trapping depths were measured by thermally stimulated method (TSC). It is found that the nanoparticle surfactant polarization can significantly influence the trapping depth of vegetable insulating oil-based nanofluids. A nanoparticle polarization model considering surfactant polarization was proposed to calculate the trapping depth of the nanofluids at different nanoparticle sizes and surfactant thicknesses. The results show the calculated values of the model are in a fairly good agreement with the experimental values.
\end{abstract}

Keywords: $\mathrm{Fe}_{3} \mathrm{O}_{4}$ nanoparticle, Nanofluids, Surfactant, Trapping depth, Model

\section{Introduction}

Vegetable insulating oils have been used as substitutes for mineral insulating oils, which are the most widely used dielectric liquids in oil-filled transformers. Recent research has shown that the thermal conductivity and breakdown voltage of insulating oils can be enhanced remarkably by adding well-dispersed nanoparticles into the oils $^{1-7}$. Magnetite nanoparticles from ferrofluids were used to prepare insulating oil-based nanofluids- - both AC and DC breakdown voltages were increased at the proper composition ${ }^{4,6}$. The $\mathrm{TiO}_{2}$ semiconductive nanoparticles also improved the dielectric breakdown performances of mineral and vegetable insulating oils ${ }^{3,5,10}$.

Electric charge carrier transportation is influenced through trapping and de-trapping processes of charge carriers in insulation materials. Traps in an insulation material can be formed by chemical defects, such as carbonyl 
groups $(\mathrm{C}=\mathrm{O})$ in vegetable insulating oils ${ }^{8}$. They can also be induced by nanoparticles in high electrical field to create trapping sites for electric charge carriers, because the dielectric constant of the nanoparticles differs from that of the continuous medium ${ }^{9}$. The trapped charge carriers generate a distribution of electric potential surrounding a trap and the trapping depth is the maximum potential. Takada et al, proposed a mathematical model to calculate the trapping depth of nanoparticles in low density polyethylene and depicted that the trapping depth depends on the size, relative dielectric constant, and shape of the nanoparticles ${ }^{9}$. Several studies ${ }^{3,5,10}$ have described a physical model revealing that nanoparticles increase the trap density and reduce the velocity of streamer propagation in mineral and vegetable oil-based nanofluids. However, these publications ignored the influences of nanoparticle surfactants on the dielectric properties of insulation nanomaterial. Nanoparticles are usually non-lipophilic, and nanoparticle surface modification is an essential approach via coating surfactant on the nanoparticles to achieve long-term dispersion stability of the oil-based nanofluids ${ }^{11-13}$. For instance, the high temperature decomposition is a very effective method of surface modification for magnetite nanoparticles to obtain stable oil-based magnetite nanofluids. Because the physical and chemical properties of the surfactant are very different from those of the nanoparticle, the surfactant may critically influence the trapping characteristics of the nanofluids in the electric field.

This letter focuses on the influence of surfactant polarization on the trapping characteristics of a vegetable oil-based nanofluid modified with $\mathrm{Fe}_{3} \mathrm{O}_{4}$ nanoparticles. Three sizes of $\mathrm{Fe}_{3} \mathrm{O}_{4}$ nanoparticles with different thickness surfactant were prepared to fabricate vegetable oil-based nanofluids. Thermally Stimulated Current (TSC) experiments investigated the effect of surfactant thickness on the charge carrier trapping characteristics of the vegetable oil-based nanofluids. A model considering surfactant polarization was proposed to calculate the trapping depth of the surface-modified nanoparticle.

\section{Experimental}

Nanofluids Preparation: The $\mathrm{Fe}_{3} \mathrm{O}_{4}$ nanoparticles were obtained by the approach of high temperature decomposition ${ }^{14}$, which produced the nanoparticles with increasing size as its reaction time increased. The insulating vegetable oil-based nanofluids were obtained in the following procedures. First, $6.48 \mathrm{~g}$ of iron (III) chloride hexahydrate was dissolved in the mixture of $48 \mathrm{~mL}$ ethanol and $84 \mathrm{~mL} \mathrm{~N}$-hexane, and the obtained solution was slowly added with $21.9 \mathrm{~g}$ sodium-oleate-vigorous through magnetic string at $60{ }^{\circ} \mathrm{C}$ for $12 \mathrm{~h}$; the solution was additionally washed three times by using deionized water at $60{ }^{\circ} \mathrm{C}$ in a separatory funnel and subsequently dried in 
vacuum at $80{ }^{\circ} \mathrm{C}$ for $24 \mathrm{~h}$ to obtain oleate precursors. Secondly, $2.1 \mathrm{~g}$ iron oleate precursor and $0.64 \mathrm{~mL}$ oleic acid were mixed in $10 \mathrm{~mL}$ octadecene followed by transferring the mixture into a three-neck-round-bottom flask and drying it at $120^{\circ} \mathrm{C}$ for 30 min under nitrogen protection to remove water and oxygen; the mixture was then heated to $320{ }^{\circ} \mathrm{C}$ for $24 \mathrm{~h}, 48 \mathrm{~h}$, and $72 \mathrm{~h}$ to realize $\mathrm{Fe}_{3} \mathrm{O}_{4}$ nanoparticles in various sizes; after cooling down to room temperature, the nanoparticles were subsequently centrifuged and washed three times with ethanol and cyclohexane before drying in air at $70{ }^{\circ} \mathrm{C}$. Thirdly, the three sizes of nanoparticles with volume concentration of $0.03 \%$ were dispersed into a type of vegetable insulating oil, the FR3 mechanized from Cargill, by ultrasonic dispersion, and they

were tagged by sample A, B, and C, respectively; before electrical characterization, three nanofluids and the FR3 were dried at $85^{\circ} \mathrm{C}$ under $50 \mathrm{~Pa}$ for $72 \mathrm{~h}$.

Material Characterization: The morphology of the $\mathrm{Fe}_{3} \mathrm{O}_{4}$ nanoparticles was characterized with a JEOL JEM-2100F high resolution transmission electron microscope (TEM). The X-ray diffraction (XRD) pattern was obtained by using a powder $\mathrm{X}$-ray diffraction meter that equipped with a rotating anode and a $\mathrm{Cu}-\mathrm{K} \alpha$ radiation source. The scan step was $0.02^{\circ}$. The AC breakdown voltage of the nanofluids was measured in accordance with the IEC 60156 standard. Trapping characteristics of the nanofluids were tested with the TSC method.

\section{Results and discussion}

Fig. 1 shows the XRD results of typical $\mathrm{Fe}_{3} \mathrm{O}_{4}$ nanoparticle according to [6] and three different sized samples were obtained by high temperature decomposition method. It can be seen from the figure, the $\mathrm{XRD}_{\mathrm{patterns}}$ of $\mathrm{Fe}_{3} \mathrm{O}_{4}$ nanoparticle near $20^{\circ}$ have a very wide amorphous peak, this is because the nanoparticles surface coated with a large number of oleic acid. The sharp peaks of different samples show reveal that the nanoparticles have high crystallinity. According to JCPDS card No. $65-3107$, the $2 \theta$ values of $30.1^{\circ}, 35.5^{\circ}, 43.1^{\circ}, 56.9^{\circ}$, and $62.6^{\circ}$ are signatures of $(220)$, (311), (400), (511) and (440) crystal face for $\mathrm{Fe}_{3} \mathrm{O}_{4}$, respectively. Therefore, these XRD patterns may be indicative of the spinel structure of the $\mathrm{Fe}_{3} \mathrm{O}_{4}$ nanoparticles. 


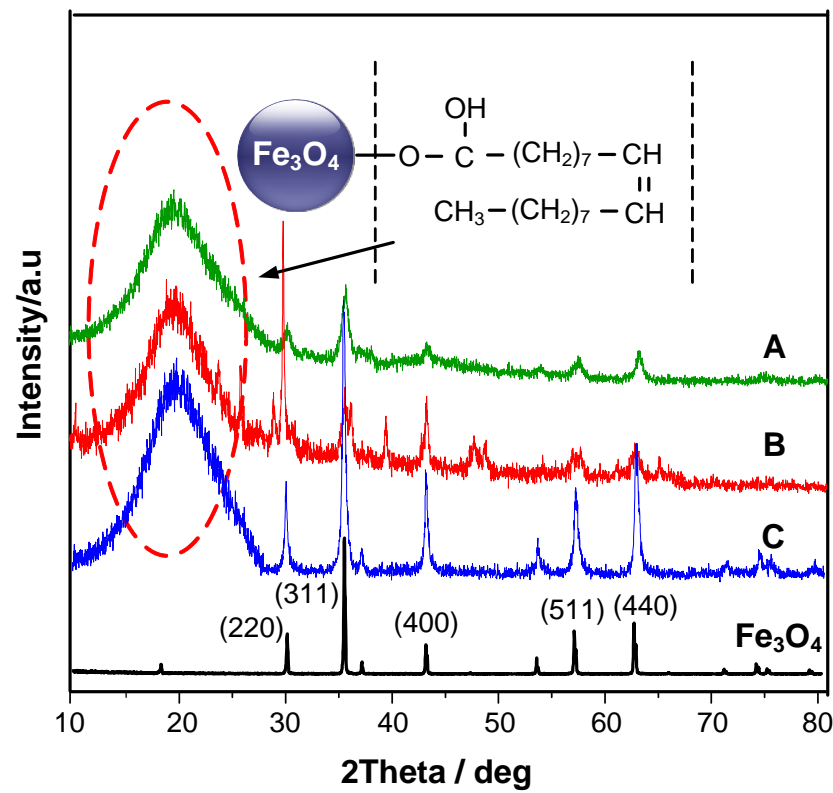

FIG. 1. XRD patterns of pure $\mathrm{Fe}_{3} \mathrm{O}_{4}$ and $\mathrm{Fe}_{3} \mathrm{O}_{4}$ nanoparticle at different reaction time: (a) 24 hours, (b) 48 hours and (c) 72 hours.

The morphologies of the three nanoparticle classes and the schematic of the core-shell nanoparticles are shown in Figure 2. The nearly spherical $\mathrm{Fe}_{3} \mathrm{O}_{4}$ nanoparticles (Figures 2a-c) are monodisperse; each nanoparticle consisting of two regions - the dark central-core is the $\mathrm{Fe}_{3} \mathrm{O}_{4}$ crystal and the surrounding layer is a low density shell of oleic acid. Covalent binding between oleic acid and $\mathrm{Fe}_{3} \mathrm{O}_{4}$ crystals prevents agglomeration of $\mathrm{Fe}_{3} \mathrm{O}_{4}$ nanoparticles and also improves the compatibility between the nanoparticles and the vegetable insulating oil. We selected two attached $\mathrm{Fe}_{3} \mathrm{O}_{4}$ nanoparticles and measured their surfactant thickness $d$ and crystal size $2 a$ (Figure 2d). The surfactant thickness $d$ is half of the distance between the crystals of the two attached nanoparticles. Figure 2 shows that the thickness of the surfactant shell changes from 1.5 to 1.4 , and $1.8 \mathrm{~nm}$; the $\mathrm{Fe}_{3} \mathrm{O}_{4}$ crystals grow from 13.7 to 23.0 and $40.7 \mathrm{~nm}$ as the reaction time increases from 24 to 72 hours. The high magnification TEM images (inset in top right) clearly show the border between surfactant shell and $\mathrm{Fe}_{3} \mathrm{O}_{4}$ crystal. Meanwhile, we define the surfactant thickness ratio as $p=d / 2 a$. It decreases as reaction time increases.

Figure 2 presents the AC breakdown voltage of the nanofluids and the FR3 oil. The measurement result of the FR3 oil is marked as the sample with nanoparticles in size of $0 \mathrm{~nm}$ added. The AC breakdown voltage of nanofluids increases as a function of nanoparticle size. That is, the AC breakdown voltage of nanofluids increases by $24.5 \%$ from $55.1 \mathrm{kV}$ for the $\mathrm{FR} 3$ fluid to $68.5 \mathrm{kV}$ for the nanofluid $\mathrm{C}$ containing $40.7 \mathrm{~nm} \mathrm{Fe}_{3} \mathrm{O}_{4}$ nanoparticles. The breakdown strength increases for nanofluids with a smaller thickness ratio of surfactant. 

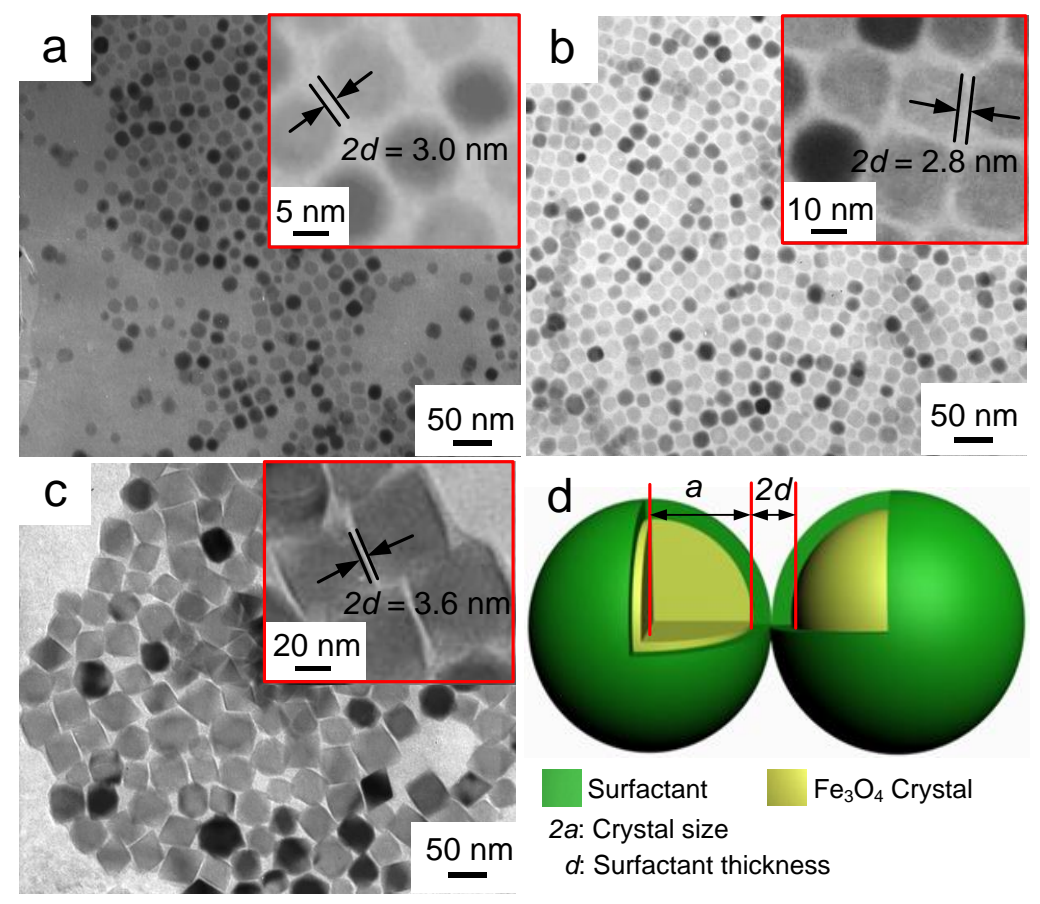

FIG. 2. TEM images of $\mathrm{Fe}_{3} \mathrm{O}_{4}$ nanoparticles at different reaction times. (a) 24 hours, (b) 48 hours, (c) 72 hours and (d) the schematic of $\mathrm{Fe}_{3} \mathrm{O}_{4}$ nanoparticle

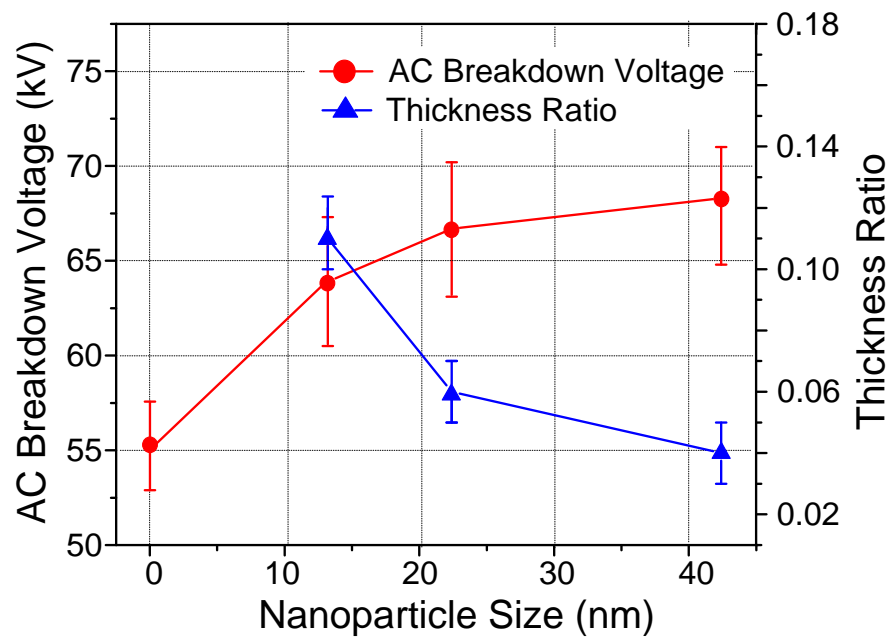

FIG. 3. Influence of nanoparticle size and thickness ratio on the AC breakdown voltage of the vegetable oil-based nanofluids.

The breakdown mechanisms of nanofluids are related to the trapping depth of the nanofluids. In figure 2, it is found that the surfactant is coated on the nanoparticle surface and the surfactant volumetric concentration is $40 \%$ over the complete nanoparticle volume (Figure 2a). The surfactant may critically influence the trapping depth of the nanofluids. In order to understand the effect of surfactant on the trapping depth of nanofluids, the trapping depth of nanofluids were measured by TSC and calculated by nanoparticle polarization model. 
The TSC curves of the pure FR3 oil and the three different sized vegetable oil-based nanofluids were tested ${ }^{5}$ and shown in Figure 4; we calculated the trapping depth ${ }^{15}$ of the FR3 oil and the three sized nanofluids equal to $0.353,0.403,0.569$, and $1.111 \mathrm{eV}$, respectively. These results indicate that the nanoparticles improve the trapping depth of the vegetable insulating oil. The trapping depth of the three nanofluid samples increases as the nanoparticle size grows up.

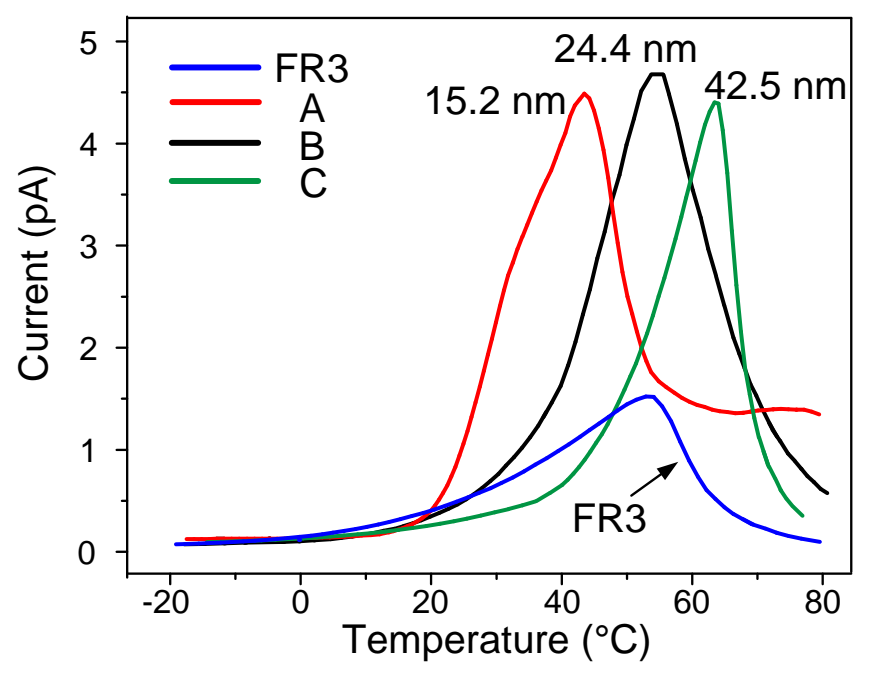

FIG. 4. The results of thermally stimulated currents in vegetable insulating oil and three sizes of nanofluids

We used the nanoparticle polarization model presented in [9] to calculate the trapping depth of the vegetable insulating oil-based nanofluids. The model supposes a spherical nanoparticle (size 2a, relative dielectric constant $\left.\varepsilon_{2}=20\right)$ in an insulation material (relative dielectric constant $\left.\varepsilon_{1}=3.2\right)$ and an external electric field $\left(E_{0}=4 \mathrm{kV} / \mathrm{mm}\right)$ applied in the direction of the x-axis. Polarization charges gather on the surface of the nanoparticle: the positive dipole surface charge is induced between the angle range $-\pi / 2<\varphi<\pi / 2$, and the negative dipole surface charge is induced between the angle range $\pi / 2<\varphi<3 \pi / 2$. The electric potential distribution, $V(r, \varphi)$, induced by the dipole surface charge density, is as:

$$
V(r, \varphi)=\frac{a E_{0}}{4 \pi}\left(\frac{\varepsilon_{2}-\varepsilon_{1}}{2 \varepsilon_{1}+\varepsilon_{2}}\right) \times \int_{-\pi}^{\pi} \int_{0}^{\pi} \frac{\sin ^{2} \theta \mathrm{d} \theta \cos \varphi \mathrm{d} \varphi}{\sqrt{1+(r / a)^{2}-2(r / a) \sin \theta \cos \left(\varphi-\varphi^{\prime}\right)}} .
$$




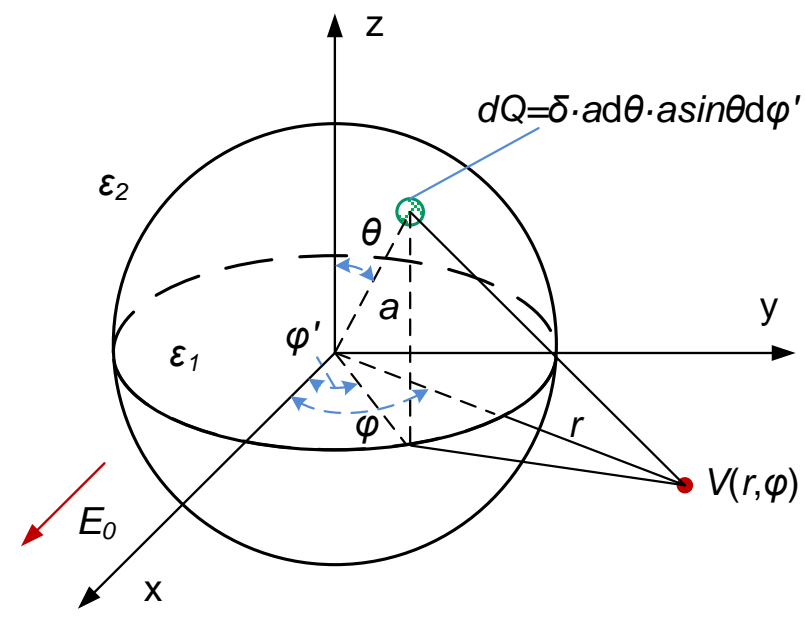

FIG. 5. Surface polarization of charged nanoparticles in a continuous dielectric field

Table I shows the trapping depth of the three sizes of nanofluids measured by the TSC and calculated by the polarization model. The calculated trapping depth of nanofluids A, B and C are larger than the measured results by $0.155,0.347$, and $0.663 \mathrm{eV}$, respectively. This indicates that the nanoparticle polarization model might not be appropriate to calculate the trapping depth of surface-modified nanoparticles. Based on our experiments results, it is believe that the surfactant can be polarized in high electric field, and significant influence the trapping depth of the nanofluids.

TABLE I. Comparison of calculated and measured trapping depths.

\begin{tabular}{ccccc}
\hline \hline Samples & $\begin{array}{c}\text { Crystal size } \\
(\mathrm{nm})\end{array}$ & $\begin{array}{c}\text { Surfactant } \\
\text { thickness }(\mathrm{nm})\end{array}$ & $\begin{array}{c}\text { TSC measured } \\
\text { trapping depth }(\mathrm{eV})\end{array}$ & $\begin{array}{c}\text { Calculated trapping } \\
\text { depth }(\mathrm{eV})\end{array}$ \\
\hline Nanofluid A & 13.7 & 1.5 & 0.403 & 0.558 \\
Nanofluid B & 23.0 & 1.4 & 0.569 & 0.916 \\
Nanofluid C & 40.7 & 1.8 & 1.111 & 1.774 \\
\hline \hline
\end{tabular}

We proposed a nanoparticle polarization model that considers surfactants to calculate the trapping depth of the nanofluids, for the purpose of investigating the influence of surfactant thickness on carrier trapping characteristics of the nanofluids. Figure 6 shows a surfactant-modified nanoparticle in the vegetable insulating oil-based nanofluid under an external electric field — both the nanoparticle and the surfactant are polarized. Positive and negative surface polarization gathers at both sides of the nanoparticle and the surfactant (Figure 6a). The surfactant polarization 
results in the electrically opposite charge gathering at the inner side of the surfactant — this partially neutralizes the $\mathrm{Fe}_{3} \mathrm{O}_{4}$ crystal surface polarization charge while the same amount of charge distributes at the outer side of the surfactant (Figure 6 b).

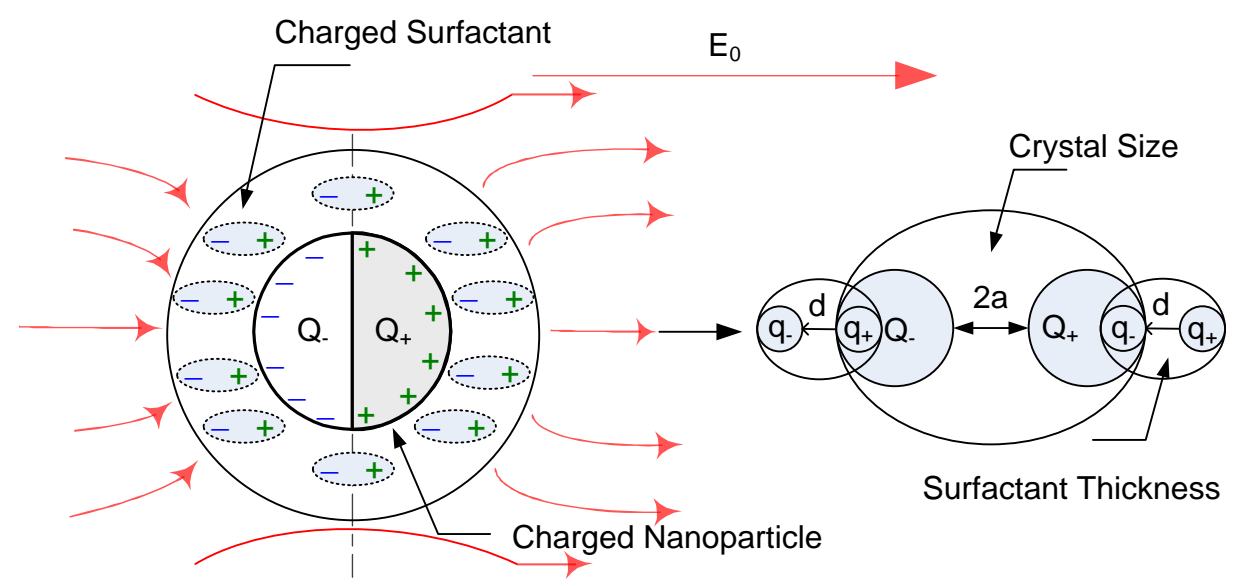

(a)

(b)

FIG. 6. Nanoparticle and surfactant polarization under external electrical field

According to nanoparticle polarization ${ }^{9}$, the $\mathrm{Fe}_{3} \mathrm{O}_{4}$ crystal surface polarization charge density that does not

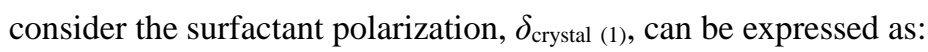

$$
\delta_{\text {crystal }(1)}=\varepsilon_{0} E_{0}\left(\frac{\varepsilon_{2}-\varepsilon_{3}}{2 \varepsilon_{3}+\varepsilon_{2}}\right) \cos \varphi \sin \theta,
$$

where $\varepsilon_{3}=2.5$ is the relative dielectric constants of oleic acid surfactant.

The outer surfactant polarization charge density $\delta_{\text {sur (outer) }}$ can be expressed as:

$$
\delta_{\text {sur (outer) }}=\varepsilon_{0} E_{0}\left(\frac{\varepsilon_{\mathrm{n}}-\varepsilon_{1}}{2 \varepsilon_{1}+\varepsilon_{\mathrm{n}}}\right) \cos \varphi \sin \theta,
$$

where $\varepsilon_{n}$ is the relative permittivities of the $\mathrm{Fe}_{3} \mathrm{O}_{4}$ nanoparticle. These can be calculated by Maxwell-Garnett formula ${ }^{16}$ :

$$
\frac{\varepsilon_{\mathrm{n}}-\varepsilon_{1}}{\varepsilon_{\mathrm{n}}+2 \varepsilon_{1}}=\varphi_{\mathrm{v}} \frac{\varepsilon_{2}-\varepsilon_{1}}{\varepsilon_{2}+2 \varepsilon_{1}}
$$

where $\varphi_{v}$ is the $\mathrm{Fe}_{3} \mathrm{O}_{4}$ crystal volumetric concentration in the $\mathrm{Fe}_{3} \mathrm{O}_{4}$ nanoparticle.

The electric potential distribution induced by the surfactant outer polarization charge density, $V_{\text {sur (out })}(r, \varphi)$, can be expressed as: 


$$
\begin{aligned}
V_{\text {sur (outer) }}(r, \varphi)= & \frac{(a+d) E_{0}}{4 \pi}\left(\frac{\varepsilon_{\mathrm{n}}-\varepsilon_{1}}{2 \varepsilon_{1}+\varepsilon_{\mathrm{n}}}\right) \\
& \times \int_{-\pi}^{\pi} \int_{0}^{\pi} \frac{\sin ^{2} \theta \mathrm{d} \theta \cos \varphi \mathrm{d} \varphi}{\sqrt{1+(r / a+d)^{2}-2(r / a+d) \sin \theta \cos \left(\varphi-\varphi^{\prime}\right)}} .
\end{aligned}
$$

As the $\mathrm{Fe}_{3} \mathrm{O}_{4}$ crystal size $2 a$ is much bigger than the surfactant thickness $d$, Eq. (5) can be simplified as:

$$
\begin{aligned}
V_{\text {sur (outer) }}(r, \varphi)= & \frac{(a+d) E_{0}}{4 \pi}\left(\frac{\varepsilon_{\mathrm{n}}-\varepsilon_{1}}{2 \varepsilon_{1}+\varepsilon_{n}}\right) \\
& \times \int_{-\pi}^{\pi} \int_{0}^{\pi} \frac{\sin ^{2} \theta \mathrm{d} \theta \cos \varphi \mathrm{d} \varphi}{\sqrt{1+(r / a)^{2}-2(r / a) \sin \theta \cos \left(\varphi-\varphi^{\prime}\right)}} .
\end{aligned}
$$

Meanwhile, the surfactant is electrically neutral, which means the quantity of the inner polarization charge equals that of the outer opposite polarization charge. Thus, the inner surfactant polarization charge density, $\delta$ sur (inner), can be expressed as:

$$
\begin{gathered}
\int_{-\pi}^{\pi} \int_{0}^{\pi} \delta_{\text {sur (outer) }}(a+d)^{2} \sin \theta \cos \varphi \mathrm{d} \varphi+\int_{-\pi}^{\pi} \int_{0}^{\pi} \delta_{\text {sur (inner) }} a^{2} \sin \theta \cos \varphi \mathrm{d} \varphi=0 \\
\delta_{\text {sur (inner) }}=\varepsilon_{0} E_{0}\left(\frac{a+d}{a}\right)^{2}\left(\frac{\varepsilon_{\mathrm{n}}-\varepsilon_{1}}{2 \varepsilon_{1}+\varepsilon_{\mathrm{n}}}\right) \sin \theta \cos \varphi
\end{gathered}
$$

Considering charge neutralization, the $\mathrm{Fe}_{3} \mathrm{O}_{4}$ crystal surface charge density that considers the surfactant polarization, $\delta_{\text {crystal (2) }}$, can be expressed as:

$$
\delta_{\text {crystal(2) }}=\delta_{\text {crystal(1) }}-\delta_{\text {sur(inner) }}=\varepsilon_{0} E_{0}\left[\left(\frac{\varepsilon_{2}-\varepsilon_{1}}{2 \varepsilon_{1}+\varepsilon_{2}}\right)-\left(\frac{a+d}{a}\right)^{2}\left(\frac{\varepsilon_{n}-\varepsilon_{1}}{2 \varepsilon_{1}+\varepsilon_{\mathrm{n}}}\right)\right] \sin \theta \cos \varphi,
$$

and the electric potential distribution induced by the $\mathrm{Fe}_{3} \mathrm{O}_{4}$ crystal surface polarization charge, $V_{\text {crystal }}(r, \varphi)$, can be expressed as:

$$
\begin{aligned}
\mathrm{V}_{\text {crystal }}(r, \varphi)= & \frac{a E_{0}}{4 \pi}\left[\left(\frac{\varepsilon_{2}-\varepsilon_{1}}{2 \varepsilon_{1}+\varepsilon_{2}}\right)-\left(\frac{a+d}{a}\right)^{2}\left(\frac{\varepsilon_{\mathrm{n}}-\varepsilon_{3}}{2 \varepsilon_{3}+\varepsilon_{\mathrm{n}}}\right)\right] \\
& \times \int_{-\pi}^{\pi} \int_{0}^{\pi} \frac{\sin ^{2} \theta \mathrm{d} \theta \cos \varphi \mathrm{d} \varphi}{\sqrt{1+(r / a)^{2}-2(r / a) \sin \theta \cos \left(\varphi-\varphi^{\prime}\right)}} .
\end{aligned}
$$

The electric potential distribution of the $\mathrm{Fe}_{3} \mathrm{O}_{4}$ nanoparticle, $V_{\text {nano }}(r, \varphi)$, is the superposition of the solutions of Eq. (6) and (10). It can be expressed as: 


$$
\begin{aligned}
\mathrm{V}_{\text {nano }}(r, \varphi)= & \frac{a E_{0}}{4 \pi}\left[\left(\frac{\varepsilon_{2}-\varepsilon_{1}}{2 \varepsilon_{1}+\varepsilon_{2}}\right)-\left(\frac{a d+d^{2}}{a^{2}}\right)\left(\frac{\varepsilon_{\mathrm{n}}-\varepsilon_{3}}{2 \varepsilon_{2}+\varepsilon_{\mathrm{n}}}\right)\right] \\
& \times \int_{-\pi}^{\pi} \int_{0}^{\pi} \frac{\sin ^{2} \theta \mathrm{d} \theta \cos \varphi \mathrm{d} \varphi}{\sqrt{1+(r / a)^{2}-2(r / a) \sin \theta \cos \left(\varphi-\varphi^{\prime}\right)}} .
\end{aligned}
$$

Figure 7 shows the trapping depth of the three sizes of nanoparticles calculated by the proposed model (model A) along the line coincident to the direction of the electric field $E_{0}=4 \mathrm{kV} / \mathrm{mm}, \theta=\pi / 2$, and $\varphi=0$. The measured TSC data are presented in Figure 7 along with calculated values using the nanoparticle polarization model without considering surfactant polarization (model B). The maximum trapping depth occurs at the surface of the nanoparticle. It increases with increasing the nanoparticle size. The calculated trapping depth of the three nanofluid samples agrees well with the TSC measurement results when model A considers surfactant polarization. Samples A and B in particular have good agreement between calculated and experimental values of trapping depth.
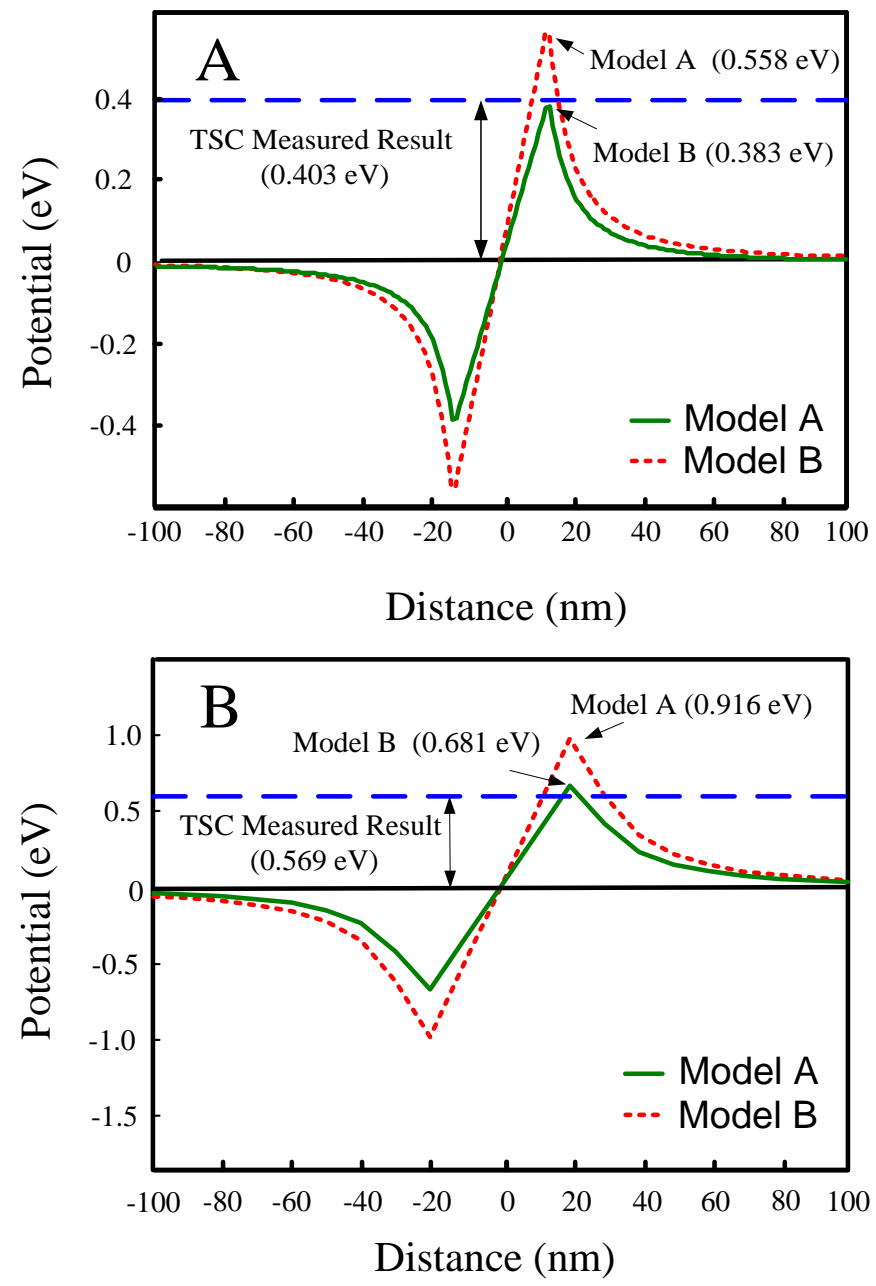


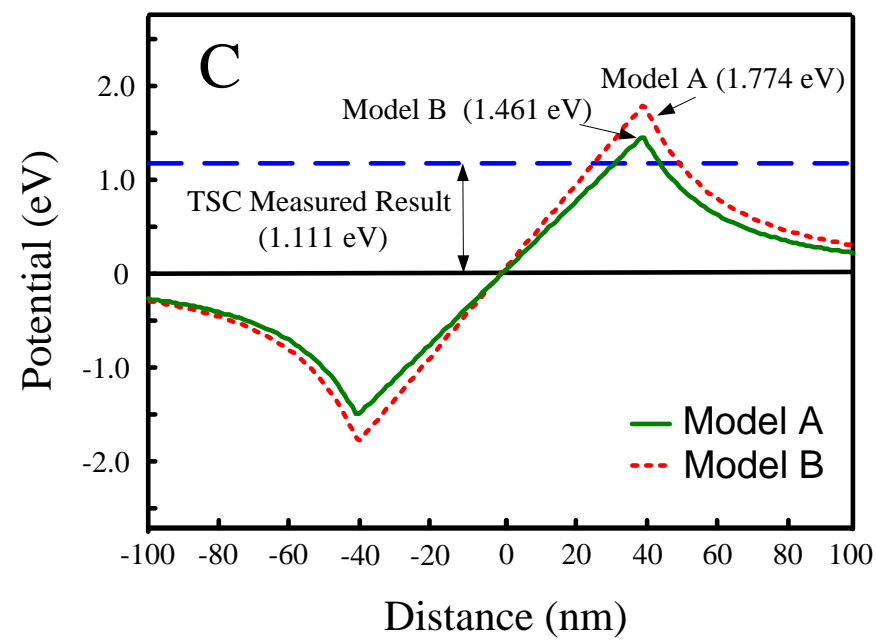

FIG. 7. Electrical potential distribution measured and calculated by models A and B of nanoparticle with (a) crystal size $2 a=13.7$ $\mathrm{nm}$ and surfactant size $d=1.5 \mathrm{~nm}$, (b) crystal size $2 a=23.0 \mathrm{~nm}$ and surfactant size $d=1.4 \mathrm{~nm}$, (c) crystal size $2 a=40.7 \mathrm{~nm}$ and surfactant size $d=1.8 \mathrm{~nm}$.

\section{Conclusion}

In conclusion, the results indicate that the surfactant can be polarized at high electric field, and significant influence the trapping depth of nanofluids. A nanoparticle polarization model that considers surfactant polarization explains the mechanisms of vegetable insulating oil-based nanofluid trapping depth, and the calculations match nicely with the values measured with TSC.

\section{Acknowledgement}

The authors acknowledge National Science Foundation of China (No.51321063 and 51377176) and the 111 Project of the Ministry of Education, China (B08036). We also appreciate the Doctoral Program of Higher Education of China (SRFDP) (20110191110017) and the funding provided by the State Key Lab of Power Equipment \& System Security and New Technology at Chongqing University (No. 2007DA10512713203).

\section{References}

${ }^{1}$ Q.Z. Xue, Phys. Lett. A 307 (2003) 313.

${ }^{2}$ L.P. Shen, H. Wangb, and M. Dong. Phys. Lett. A 376 (2012) 1053. 
${ }^{3}$ Y. Z. Lv, Y. F. Du, and C. R. Li, Appl. Phys. Lett. 102 (2013) 132902.

${ }^{4}$ J. G. Hwang, M. Zahn, and F. M. O’Sullivan, J. Appl. Phys. 107 (2010) 014310.

${ }^{5}$ Y. F. Du, Y. Z. Lv, C. R. Li, M. T. Chen, and Y. X. Zhong, J. Appl. Phys. 111 (2012) 124322.

${ }^{6}$ J. Li, Z. Zhang, P.Zou, S. Grzybowski, and M. Zahn, IEEE Electr. Insul. Mag. 28 (2012) 43.

${ }^{7}$ X. B Wang, Z. H Wang, X. J. Hao, Appl. Phys. Express 7 (2014) 083002.

${ }^{8}$ M. Meunier, N. Quirke, and A. Aslanides, J. Chem. Phys. 115 (2001) 2866.

${ }^{9}$ T. Takada, Y.Hayase, and Y. Tanaka, IEEE Trans. Dielect. Electr. Insul. 11 (2004) 763.

${ }^{10}$ Y. F. Du, Y. Z. Lv, C. R. Li and B. Qi, J. Appl. Phys. 102 (2013) 132902.

${ }^{11}$ K. D. Kim, S. S. Kim, Y. H. Choa, and H. T. Kim, J. Ind. Eng. Chem. 13 (2007) 1137.

${ }^{12}$ K. M. Kamruzzaman Selim, Y. S. Ha, S. J. Kim, and Y. Chang, Biomaterials. 28 (2007) 710.

${ }^{13}$ T. Tsai, L. Kuo, P. Chen, D. Lee, and C. Yang, Sensors, 10 (2010) 8161.

${ }^{14}$ V. M. Kovalenko, M. I. Bodnarchuk, and R. T. Lechner, J. Am. Chem. Soc. 129 (2007) 6352.

${ }^{15}$ Q. Q. Lei, X. Wang and Y. Fan, J. Appl. Phys. 72 (1992) 4254.

${ }^{16}$ J. Mao, M. Dong, M. Ren and X. Z. Wu, J. Appl. Phys. 113 (2013) 204103. 